\title{
Research on Novel Psychological Measurement Methodology based on Computer Assisted Approach
}

\author{
Zhang Hao ${ }^{1}$ \\ ${ }^{1}$ Guizhou Normal University, \\ GuiYang City,GuiZhou Province 550001 China
}

\begin{abstract}
In this paper, we conduct survey on the novel psychological measurement methodology based on computer assisted approach. Data acquisition and mining can forecast the future trend of the psychological and behavior, it is found that of the novel, effective and fully able to understand the data model of a kind of method. Our designed system takes the usage of computer and calculation system to better simulate the analyzing process. The numerical simulation shows the effectiveness and robustness of the system and the prospect is set in the final part.
\end{abstract}

\section{Keywords: Psychological Measurement;} Computer Assisted; System Development.

\section{Introduction}

In the field of psychology, computer technology has also been dramatic psychological measurement field, changed the traditional form of paper and pencil tests, people began to use computers to participate in the whole measurement process, including from test preparation to the implementation of the test, and the subsequent score, report and explain. For decades, due to the development and popularization of computer technology and its obvious advantages in actual use, accelerate the computer testing technology in the field of psychology measurement scale and the application of questionnaire. Computer advantages such as rapid, accurate and informative in the professional personnel selection, clinical psychological counseling, psychological training, teaching plays an important role in scientific research, etc. With the deepening development of psychometrics, it has been widely used in selecting talents, vocational guidance, according to their aptitude, mental deficiency and the early diagnosis of mental illness. At present, mental measurement scale, has introduced from abroad and modified already, also have psychological workers in China compiled by themselves. Although they are both effective psychological measurement tool, but if you do not pay attention to in the process of testing the possible influencing factors of measuring reliability and validity of control, then the experimenter the authenticity of measuring results is questionable. Another explanation for scale scores will also affect the scientific nature of psychological measurement and seriousness. Clinical psychological assessment is the important tool of psychological consultant to understand visitors, is also a clinical doctor understand patients psychological state of the important methods for the body disease. But how to accurately and efficiently measure visitors or body disease patients psychological status, is suspends in front of psychological measurement professionals an important subject. In relatively tense doctor-patient relationship, if the doctor can according to the patient's overall psychological status and humanistic diagnosis and treatment plan, will be to a great extent and promote the patient recover as soon as possible, also is advantageous to the construction of a harmonious doctor-patient relationship [1].

Computer assisted methodology is becoming more and more popular in the research community. In the paper, we review and analyze the novel psychological measurement methodology based on computer assisted 
approach. Computer through the call in question bank project, to match the subjects ability to correct estimate of the subjects ability continuously, if the subjects right of the current project, a project will be harder to call, and vice versa. When test to reach the required standard, computer stop calling topic and stop the test. Traditional test, the test length is fixed, but the test precision is changing. In general, most of the traditional test project is medium difficulty, so the estimate of the secondary ability subject error is smaller. In the figure one, we illustrate the sample library for the computer assisted testing system. In the following sections, we will discuss and analyze the system in theoretical and numerical in detail.
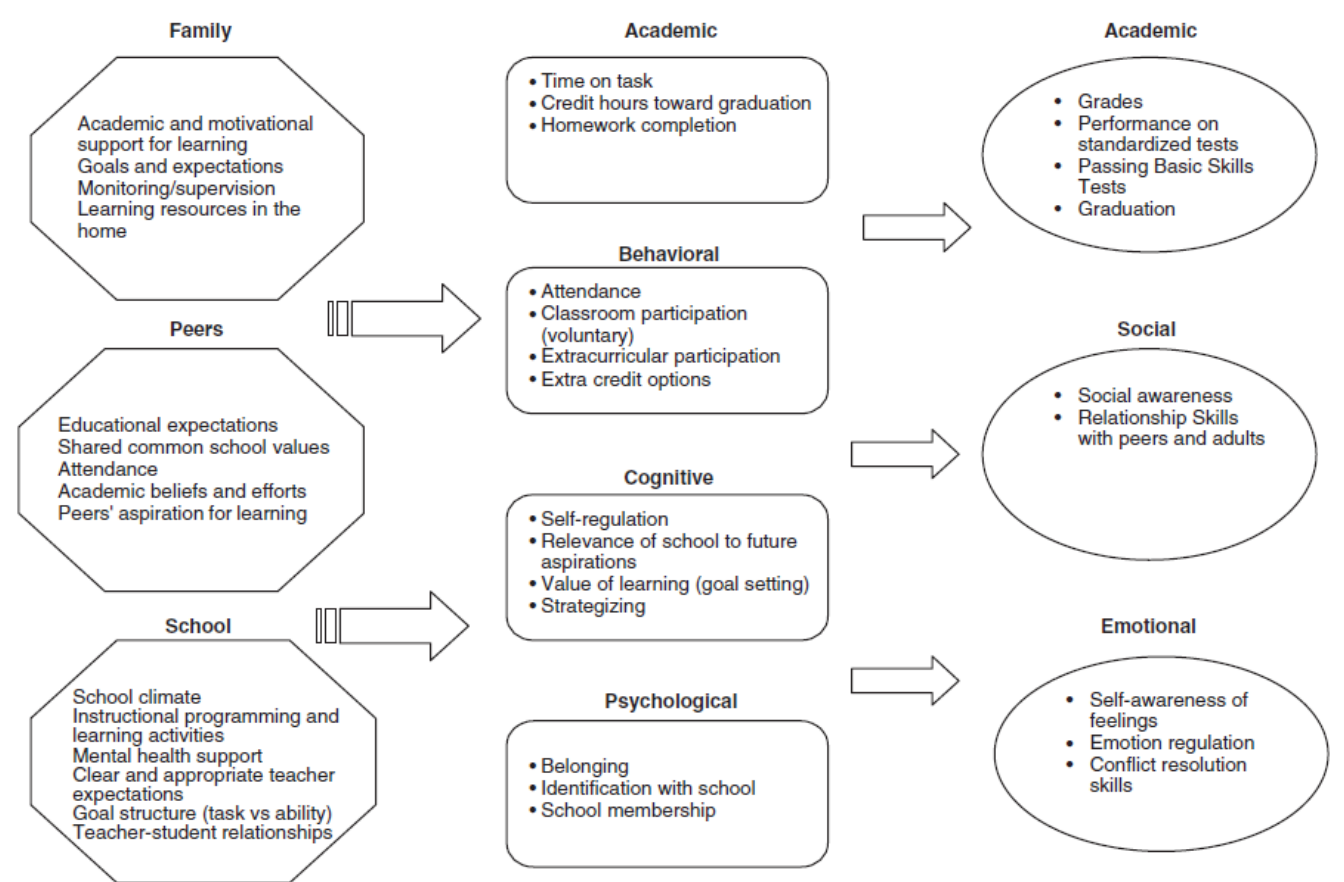

Fig 1. The Sample Library for the Computer Assisted Testing System

\section{The Proposed Methodology and System Design}

The General Information on Psychological Measurement. There have been several big and important mainstream psychological research methods [2]. Tended to sociology, psychology and anthropology of the tendency of social psychology hold great differences on the way. Social studies could not put aside due to a collection of individuals who form the social psychological research, and by the members of the community mentality and social culture shown by the study of the various kinds of faces, most scholars usually is mainly using depth interview method to obtain subjects related information, finally on the basis of summary of qualitative conclusions. The measurement of organizational identification, in fact, is the use of scale measurement method, measurable way to use the content of the measurement for corresponding implementation measure, the last expression in the number of research results. Because of organizational identification is involved in the mindset of organization members and organizational culture phenomenon, adopt the method of general measure the subjectivity problem exists uncertainty of confusion. Therefore, to consider such as what should be measured in the measurement, how to set its operationalization and a series of problem such as measurement is correct. In social life, always for people place praise highly idealized behavior, 
which makes people to oneself, also to others and often performed according to the standard of the ideal. They may be because of their ability to actually reach ideal standard requirement and trying to hide my own shortcomings, may also be in some ways do not conform to the idealized standard and conceal relevant respects, it is also possible that some things performance process may not be as grandiose as show people or extremely skilled and hide process hard even is unseemly, and so on. With the development of the computer, enables the mind as an information processing system research, converts sensibility analysis to intuitive information statistics, can enable psychologists to find out more methods to directly observe things, as well as between stimulus and response variables, such as: attention, thinking and decision variables involved. By idealized performances, viewers saw in the front desk and are completely meet the requirements of the society for the performers' idealized action. The aided measurement system can achieve the most suitable subjects entry test, even though different subjects complete title is different, the results are comparable. Moreover, due to the difficulty of the all questions are unified in the same dimensions. Real-time assessment shall be conducted by computer program. Extraction is suitable for the topic of the subjects for testing. Unlike ability test, the test due to the specific traits by extensive variety of forms, test preparation, test is based on the theoretical basis of different [3]. So you can't like ability test to its unidimensional test. In the next section, we will discuss the system building.
The Computer Assisted Approach. In this paper, the test method is adopted for the design of the system, make use of these tests, we can measure an individual's intelligence, special ability and cognitive strategies, also can learn more about a region, a group, someone in the cognitive characteristic, can understand the rule of cognitive development in the process of growth and maturity which can understand different cultures, the question of differences between countries, between different ethnic groups and the differences between people of different industries, the test method on the understanding of individual and group uses a very wide range. The main use of the principle of psychological statistics test method or psychological statistics is the study of the psychological experiment investigation how to collecting, sorting and analyzing digital data, according to these data information passed by, and how to make a scientific reasoning branches of applied statistics. Literature describing statistical research how to experiment or investigation of a large number of data reduction as representative figures, make its can objectively and comprehensively reflect the whole picture of this set of data, the information provided by the fully revealed, for further statistical analysis and inference. The realization of the measurement for each measurement personnel should as far as possible concise, psychological measurement can't to measure personnel's psychological pressure, and also make sure the topic of measurement to measure could refer to the actual situation of the measurer [4]. The core code block for the system is shown in the figure two. 


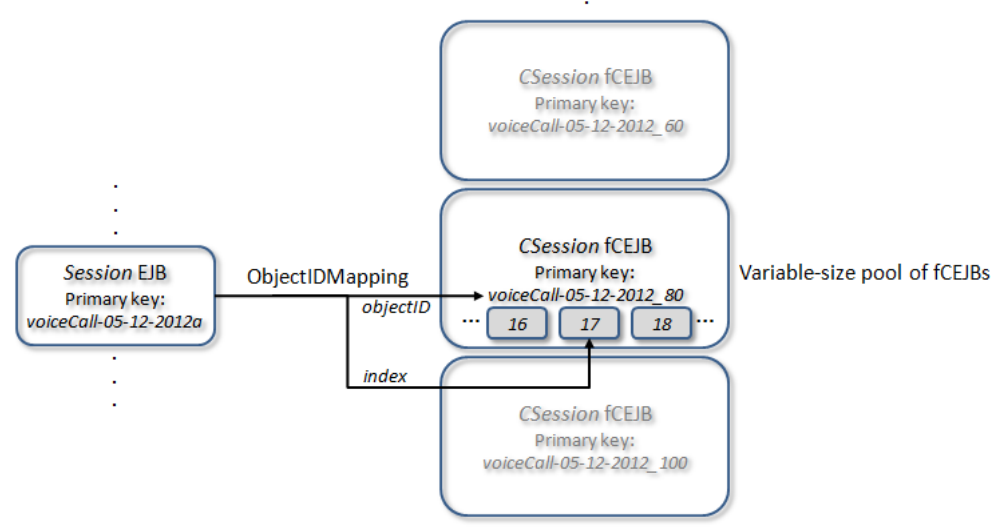

Fig 2. The Sample Core Code Block for the System

\section{The System Combination and the} Performance. Data acquisition and mining can forecast the future trend of the psychological and behavior, it is found that of the novel, effective and fully able to understand the data model of a kind of method. It combined with statistics and computing technology, from a large number of data set to obtain useful patterns, thus produce set of guiding rules, these rules are for data in the database properties, effective description of the object set, thus well support professionals or decision analysis to provide decision support system analysis. Question bank construction should pay attention to the test information function matching and test purposes. Expressing ability test information function estimation is accurate. Computer can be carried out on the test score and generate a report immediately as a result, the traditional paper and pencil test at the end of the test, need artificial scores and record the results, and then according to the norm standard score. This process complicated time-consuming and wasted a lot of manpower and related material resources. Through the corresponding computer software and hardware system can not only save the individual test results and interpretation process. Developed by groups such as measuring system is centralized, will test its unique batch result is greatly convenient for test users. It is defined as the inverse square of the error of measurement. Test and the higher the amount of information to the subjects corresponding ability to estimate the accuracy is higher. If the test is a standard reference test, according to a certain score divided subjects into pass and fail, so to score in the demarcation point can accurately distinguish the ability of subjects, namely at the line need more information, to both ends is the estimation precision of the test values do not require. If the research form conforms to the requirement of unidimensional and monotonicity, application of double parameters logistic model effect is better, if the study scale in line with the unidimensional sex but they do not conform to the requirements of the monotonicity. To continue the test revision is a kind of actual measured and the experimental test completely together with test and development, continuous to a system. The revision of the test directly implied in the measurement process itself, the new project based on the old program for the calibration, as long as meet certain standards, project can be automatically added to the question bank. With the aid of related software, scale, the researchers can easily call test result and the data record, edit, modify, and add or delete some test can merge the data on the other computer. In preparation and revision test, often need to constant modulus according to calculation, reliability and validity 
of inspection and analysis project, involved in the complex statistical analysis by the corresponding program on the computer or software can be done easily. Because in such revision of the system, need to get a lot of data quickly, so a centralized computerized system is very important. In the figure three, we illustrate the system performance in detail.

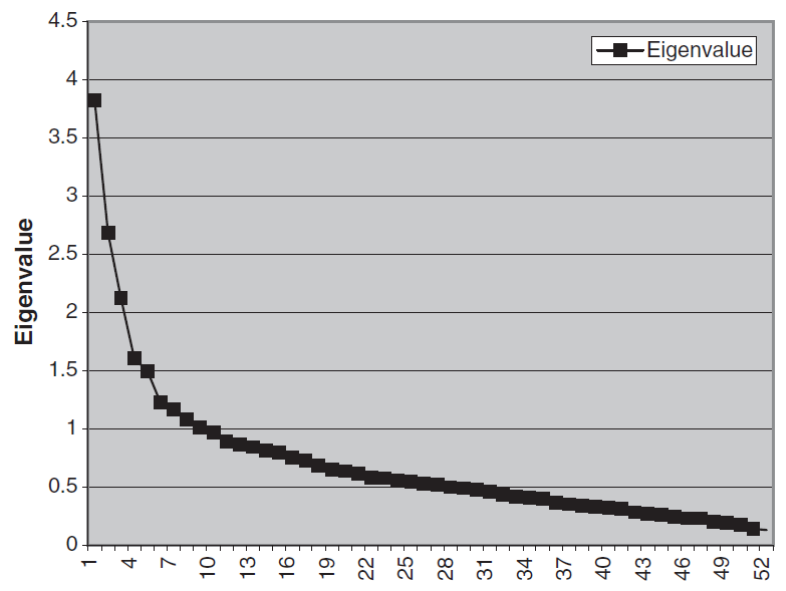

Fig 3. The System Performance for the Psychological Measurement

\section{Conclusion}

In this paper, we conduct survey on the novel psychological measurement methodology based on computer assisted approach. Computer advantages such as rapid, accurate and informative in the professional personnel selection, clinical psychological counseling, psychological training, teaching plays an important role in scientific research. In our designed system, the new project based on the old program for the calibration, as long as meet certain standards, project can be automatically added to the question bank. In the experimental part, we show that the computer assisted method is effective.

\section{References}

[1] McArdle J J, Hamagami F, Bautista R, et al. Multilevel Factor Analyses of Family Data from the Hawai'i Family Study of Cognition.[J]. Educational \& Psychological Measurement, 2014.

[2] Sideridis G, Simos P, Papanicolaou A, et al. Using Structural Equation Modeling to Assess Functional Connectivity in the Brain: Power and Sample Size Considerations.[J]. Educational \& Psychological Measurement, 2014, 74(5):733-758.

[3] Sliter K A, Zickar M J. An IRT Examination of the Psychometric Functioning of Negatively Worded Personality Items.[J]. Educational \& Psychological Measurement, 2014, 74(2):214-226.

[4] Xiao-feng, L., Chen-chen, R., Xing-xing, P., Lun-lai, W., \& Economics, M. O. (2014). Study on psychological measurement system of university teachers' salary discount. Operations Research \& Management Science. 